\title{
基于表面增强拉曼光谱的苹果毒死蜱残留无损检测方法
}

\author{
翟晨彭彦昆李永玉 ${ }^{*}$ 徐田锋 \\ (中国农业大学工学院 北京 100083)
}

\begin{abstract}
摘要 对表面增强拉曼光谱(Surface Enhanced Raman Spectroscopy, SERS)技术在苹果毒死蜱残留量无损快速准确检测 中的应用进行了研究. 以银溶胶作为表面增强剂, 采用实验室搭建的拉曼光谱系统, 无损地采集苹果样品拉曼光谱. 分别采用二阶导数法(Secondary Derivative Transformation, SD)和极小极大值自适应缩放法(Min-max Signal Adaptive Zooming, MSAZ)扣除所得拉曼光谱的荧光背景以消除样品和环境的干扰，从而提高检测的准确度. 对获取光谱后的苹 果样品采用标准理化方法检测其毒死蜱含量, 在最优条件下, 建立拉曼光谱与 $15.52 \sim 0.064 \mathrm{mg} / \mathrm{kg}$ 范围内毒死蜱含量 的线性关系, 结果表明毒死蜱的两个主要特征峰强度和其浓度呈良好的线性关系, 预测集的相关系数为 0.969 , 预测均 方根误差为 $1.24 \mathrm{mg} / \mathrm{kg}$. 本研究所开发的方法为果蔬安全品质的无损快速检测提供了一种新思路.

关键词 表面增强拉曼光谱; 银溶胶; 毒死蜱; 无损检测; 苹果
\end{abstract}

\section{Nondestructive Detection of Chlorpyrifos in Apples Based on Surface Enhanced Raman Scattering}

\author{
Zhai, Chen Peng, Yankun Li, Yongyu* Xu, Tianfeng \\ (College of Engineering, China Agricultural University, Beijing 100083)
}

\begin{abstract}
A new non-destructive, rapid and accurate surface-enhanced Raman spectroscopy (SERS) assay method using silver colloidal nanoparticles for the detection of chlorpyrifos pesticides in apples is developed and optimized. Silver colloidal nanoparticles are paperad by reduction of silver nitrate with hydroxylamine hydrochloride and investigated using scanning electron microscope and visible-ultraviolet spectroscopy. Trace amounts of acetone, silver colloid, and nitric acid are dropped onto apple samples and air exposed for $20 \mathrm{~s}$. The SERS spectra are collected non-destructively from the apple sample with a self-developed Raman system. The relative standard deviation (RSD) is calculated to evaluate the stability of the present method, including the stability of silver colloid and the stability of the Raman system, which indicates that the proposed strategy has good stability. Secondary derivative transformation and min-max signal adaptive zooming method are used to remove the fluorescence background for eliminating the environmental impact and improving the accuracy of SERS results. Raman spectral signals are collected from 30 points on each sample with $450 \mathrm{~mW}$ laser power and $3 \mathrm{~s}$ exposure time. Chlorpyrifos concentrations in 41 samples are determined with gas chromatography after SERS spectra taken. The characteristic peaks of chlorpyrifos at $621 \mathrm{~cm}^{-1}$ and $680 \mathrm{~cm}^{-1}$ are visible even at the concentration of $0.064 \mathrm{mg} / \mathrm{kg}$. Under optimal conditions, linear regression models are established between the SERS signal and the chlorpyrifos concentrations in the range of $15.52 \sim 0.064 \mathrm{mg} / \mathrm{kg}$. There are good linear relationships between the concentrations of chlorpyrifos pesticides in apples and the Raman intensities of its two major characteristic peaks, for the best of which the correlation coefficient of prediction $\left(R_{\mathrm{p}}\right)$ is 0.969 and the root mean square error of prediction (RMSEP) is $1.24 \mathrm{mg} / \mathrm{kg}$. The present study provides a potential method for the rapid detection of pesticides which can help to strengthen the safety and quality of our food supplies.
\end{abstract}

Keywords surface-enhanced Raman spectroscopy; silver colloid; chlorpyrifos; nondestructive detection; apple

\section{1 引言}

毒死蜱(Chlorpyrifos) 是一种高效的有机磷类杀虫 剂, 可抑制害虫体内胆碱酯酶活性, 在苹果种植过程中 应用广泛 ${ }^{[1]}$. 但是过量地使用毒死蜱, 会对生态环境和 人类健康产生严重的危害 ${ }^{[2]}$. 传统的农药残留检测方法 主要有气相法、液相法和质谱法等 ${ }^{[3,4]}$, 虽然其具有精度 高和检测限低等优点, 但是这些方法采用的仪器体积
大, 样品前处理繁琐, 仪器操作较为复杂, 很难作为一 种实时、快速, 可用于日常生活中果蔬品质检测的手段. 因此寻找一种简便、有效、快速的农药残留定量检测方 法极其重要.

利用光谱手段对果蔬的品质安全进行检测是近几 年的研究热点 ${ }^{[5,6]}$. 光谱检测方法具有灵敏度高、样品前 处理简单等优势, 将其应用于农畜产品的品质检测具有 重要意义. 拉曼光谱属于分子振动光谱, 不同的化学键

*E-mail: yyli@cau.edu.cn

Received May 11, 2015; published July 20, 2015.

Supporting information for this article is available free of charge via the Internet at http://sioc-journal.cn.

Project supported by the National Science and Technology Support Program (No. 2014BAD04B05).

项目受国家科技支撑项目(No. 2014BAD04B05)资助. 
在拉曼光谱中对应着不同的波数区域 ${ }^{[7]}$, 拉曼光谱检测 样品无需前处理, 可直接测定, 保证了样品信息的完整 性. 但是拉曼散射效应是个非常弱的过程, 一般其光强 仅约为入射光强的 $10^{-10}$, 所以拉曼信号比较弱 ${ }^{[8]}$. 表面 增强拉曼光谱(Surface Enhanced Raman Spectroscopy, SERS)方法能显著提高拉曼信号的强度, 可在分子水平 上给出丰富的结构信息, 因此采用 SERS 技术检测化学 成分成为近几年的研究热点 ${ }^{[9]}$. 拉曼光谱表面增强的效 果与表面增强剂的种类密切相关, 其中以金和银的纳米 粒子效果最好, 相比金纳米粒子, 银纳米具有较强的表 面等离子共振效应(SPR) ${ }^{[10]}$, 其表面增强效果更显著, 其增强系数为金纳米粒子的 10 至 100 倍, 甚至更高 ${ }^{[11]}$, 本文中以盐酸着弪胺还原法制备的银溶胶, 其制备过程简 单, 无需加热, 可直接用于拉曼信号的增强, 且具有较 好的稳定性.

近几年, 利用表面增强技术对果蔬表面的农药残留

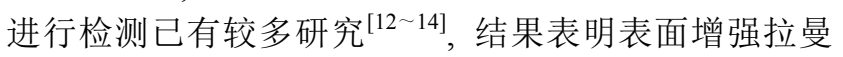
技术能够显著降低农药的检出限, 但是这些方法在扫描 拉曼光谱前, 需要对样品进行一定的前处理, 例如将样 品打碎或者采用有机溶剂拭擦样品表面提取农药. 而本 研究基于实验室自行搭建的拉曼光谱系统进行检测 ${ }^{[15]}$, 通过控制置样台的位置, 可实现对完整样品的无损检 测, 所采用的表面增强剂银溶胶只需微量滴涂到样品表 面, 不需对样品进行破坏处理, 保证了样品的完整性.

本文以银溶胶作为表面增强剂, 对苹果中的毒死蜱 残留量进行检测, 在苹果表面进行多点扫描采集拉曼光 谱后, 采用二阶导数法(Secondary Derivative Transformation, SD) 和极小极大值自适应缩放法 ${ }^{[16]}$ (Min-max Signal Adaptive Zooming, MSAZ)扣除光谱的苂光背景, 然后建立苹果样品中毒死蜱含量与拉曼光谱的数学模 型.

\section{2 结果与讨论}

\section{1 银溶胶的表征}

对制备的银溶胶进行扫描电子显微镜(SEM)和可 见-紫外吸收光谱分析, 图 1a 为银溶胶的 SEM 图像, 纳 米粒子分布均匀, 粒径大小为 $100 \sim 150 \mathrm{~nm}$. 图 $1 \mathrm{~b}$ 为通 过离心浓缩后的银溶胶 SEM 图像, 粒子体积密度变大, 但是并未发生团聚, 图 1c 为与农药混合后的银溶胶 SEM 图像, 此时具有较为明显的团聚现象, 溶液由灰色 变成灰黑色. 图 $1 \mathrm{~d}$ 中曲线 1 为银溶胶的紫外可见光谱, 该银胶的最大表面等离子体共振峰 $\lambda_{\text {max }}$ 为 $423 \mathrm{~nm}^{[17]}$, 曲 线 2 为与农药混合后的银溶胶的紫外可见光谱, 相同浓 度下, 吸收峰强度下降很多, 说明银溶胶发生了团聚, 团聚后粒子之间产生耦合作用, 增加了 “热点” 数, 待 测物分子在银溶胶聚沉过程中更容易吸附到 “热点”上, 从而使 SERS 信号得到增强 ${ }^{[18,19]}$.
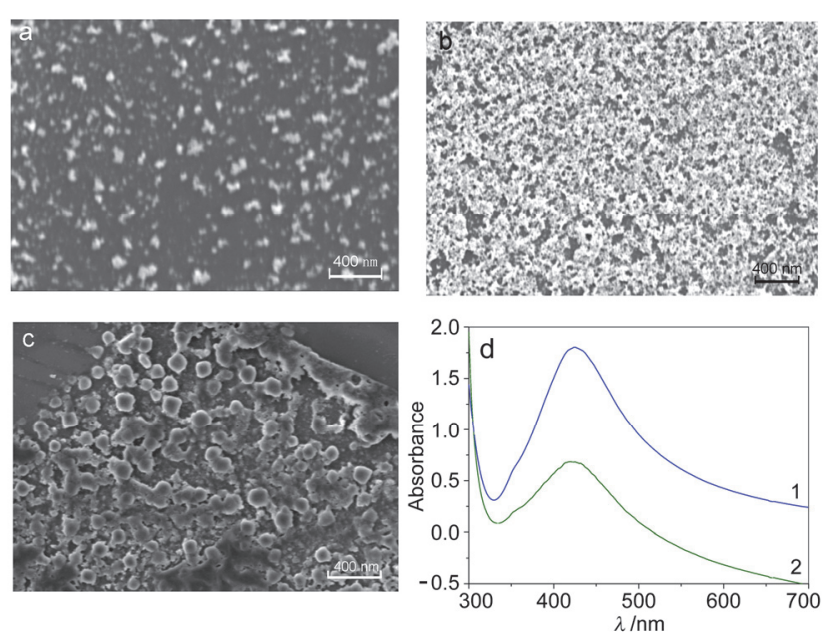

图 1 银溶胶(a), 浓缩银溶胶(b)和与农药反应后的银溶胶(c)的 SEM 图像; (d)银溶胶(1)和银溶胶与农药反应后(2)的紫外-可见分光光谱曲 线

Figure 1 SEM micrographs of silver colloid (a), concentrated silver colloid (b) and silver colloid after reaction with pesticides (c); (d) UV-vis absorption spectra of silver colloid (1) and silver colloid after reaction with pesticides (2)

\section{2 丙酮和硝酸对 SERS 的影响}

在扫描拉曼光谱之前, 需要在样品上滴加表面增强 剂, 通过多次试验, 直接将银溶胶滴涂于样品表皮，表 面增强效果不明显, 如图 2a. 推测其原因是银溶胶为水 溶液, 毒死蜱在水中的溶解度较低, 农药分子吸附于果 皮中, 直接滴加银溶胶, 并不能使毒死蜱分子充分与银 纳米粒子混合. 因此, 需要在滴加银溶胶之前滴涂丙酮 溶液, 见图 $2 b$, 使毒死蜱分子溶解分散于丙酮溶液中. 图 2c 为滴加银溶胶, 此时进行拉曼扫描, 已有比较明显 的表面增强现象. 最后滴加硝酸溶液(图 2d), 其拉曼表 面增强现象更加明显, 采用 Leopold 法制备银溶胶, 得 到的银溶胶为负电荷, 滴加硝酸, 使银溶胶附近的氢离 子 $\left(\mathrm{H}^{+}\right)$数目增多, 由于农药粒子带有负电荷, 更容易吸 附在银溶胶附近, 当待测分子吸附或者非常靠近纳米结 构时, 其拉曼信号会显著增强 ${ }^{[20]}$, 硝酸的加入进一步缩 短了农药分子与银纳米粒子的距离, 因此拉曼信号得到 增强, 但是过量加入硝酸溶液, 会导致拉曼峰显著降低, 因为过多的氢离子与农药分子产生竞争作用, 使农药分 子与银纳米粒子之间的吸附数量减少, 导致农药的拉曼 信号变弱 ${ }^{[21]}$.

\section{3 银溶胶的稳定性}

对试验的稳定性进行分析，从两方面进行探讨，一 是银溶胶的储存稳定性, 即其表面增强效果是否随着储 存时间的延长而发生变化, 二是系统以及试验方法的稳 定性, 即在相同条件下重复采集的样品光谱的峰强度是 否稳定. 


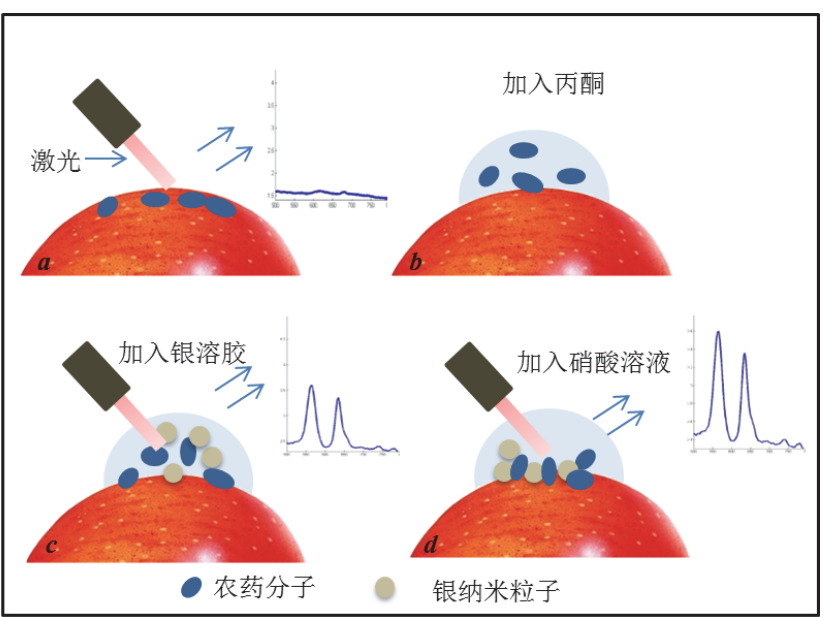

图 2 SERS 示意图

Figure 2 Schematic diagram of SERS experiments

为测试银溶胶的稳定性, 将制备好的银溶胶在 $4{ }^{\circ} \mathrm{C}$ 环境下储存, 从第 1 天至第 7 天连续每天取相同体 积的银溶胶对相同条件下的苹果样品(每天准备 10 组样 品, 其农药含量相同, 实验步骤相同)进行检测, 平均光 谱图见图 3, 毒死蜱的拉曼特征峰 $348 \mathrm{~cm}^{-1}(\mathrm{C}-\mathrm{C}$ 伸 缩)、 $535 \mathrm{~cm}^{-1}(\mathrm{P}-\mathrm{O}$ 伸缩 $) 、 568 \mathrm{~cm}^{-1}(\mathrm{C}-\mathrm{Cl}$ 伸缩 $) 、 621$ $\mathrm{cm}^{-1}(\mathrm{P}=\mathrm{S}$ 伸缩 $) 、 680 \mathrm{~cm}^{-1}(\mathrm{C}-\mathrm{Cl}$ 伸缩 $)$ 和 $1099(\mathrm{P}-\mathrm{O}$ $-\mathrm{R}$ 伸缩 ${ }^{[22]}$ 十分明显, 以 621 和 $680 \mathrm{~cm}^{-1}$ 处两个特征峰 的峰值进行比较, 在 7 天的测试过程中, 拉曼峰值波动 较小, 其图 3 中小图显示了这两个特征峰在 7 天中的峰 值变化, 621 和 $680 \mathrm{~cm}^{-1}$ 处峰强度的相对标准偏差 (Relative Standard Deviation, RSD) 分别为 4.1\%和 3.7\%. 对搭建的拉曼系统以及试验方法的稳定性进行测试, 将 苹果皮切成相同面积的小块, 均匀涂上浓度相同的毒死 蜱溶液, 采用相同的方法进行表面增强实验, 一共测试 50 块样品, 得到 50 条光谱曲线, 如图 4, 特征峰 348, 621,680 和 $1099 \mathrm{~cm}^{-1}$ 处峰值的 RSD 分别为 $7.8 \%, 5.3 \%$, $4.6 \%$ 和 $10.2 \%$, 均具有较好的重复性. 因此, 采用盐酸 羟胺还原法制备的银溶胶具有较好的稳定性, 相应的实 验装置和方法具备较好的实际应用潜力.

\section{4 光谱的预处理}

采集拉曼光谱时, 光谱系统、外界环境以及待测样 品都具有不稳定性, 因而造成较强烈的苂光背景, 导致 了拉曼信号分析时数据偏差的产生. 为了去除荧光背 景, 本研究采用二阶导数法(SD)和极小极大值自适应缩 放法(MSAZ)进行光谱数据的预处理. 二阶导数法是一 种常用的扣除荧光背景的方法, 能够有效地分辨重叠 峰 ${ }^{[23]}$. 极小极大值自适应缩放法是一种新型的数据处 理方法, 可以识别信号较小的特征峰 ${ }^{[16]}$. 光谱处理效果 如图 5 所示, 图 $5 \mathrm{a}$ 为毒死蜱含量为 $15.43 \mathrm{mg} / \mathrm{kg}$ 和 1.44 $\mathrm{mg} / \mathrm{kg}$ 的苹果样品的原始拉曼光谱图, 图 $5 b$ 为 $\mathrm{SD}$ 处理

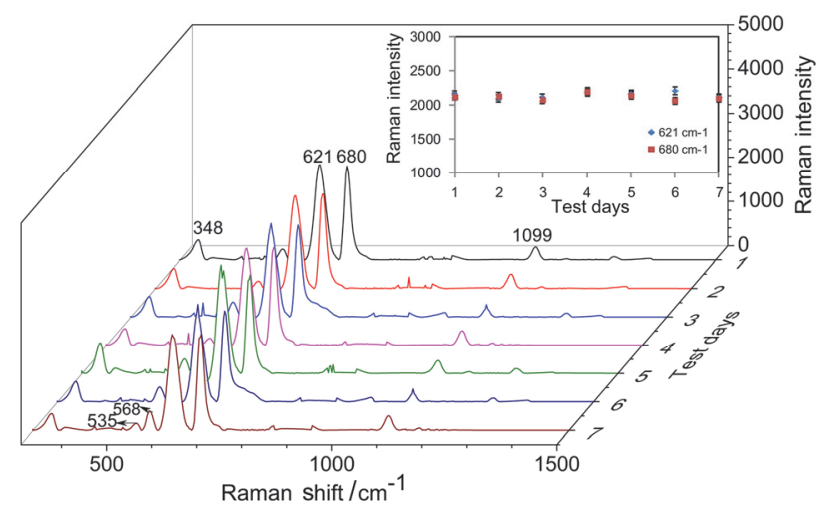

图 3 储存 $1 \sim 7$ 天的银溶胶在毒死蜱含量相同的苹果样品上产生的 SERS 谱图. 内图: $621 \mathrm{~cm}^{-1}$ 和 $680 \mathrm{~cm}^{-1}$ 处峰强度随银溶胶储存时间的 变化

Figure 3 SERS spectra of apple samples added with the same concentration of chlorpyrifos at different storage period from $1 \mathrm{~d}$ to $7 \mathrm{~d}$. Inserted image: influence of storage on peak intensities of Chlorpyrifos at 621 $\mathrm{cm}^{-1}$ and $680 \mathrm{~cm}^{-1}$

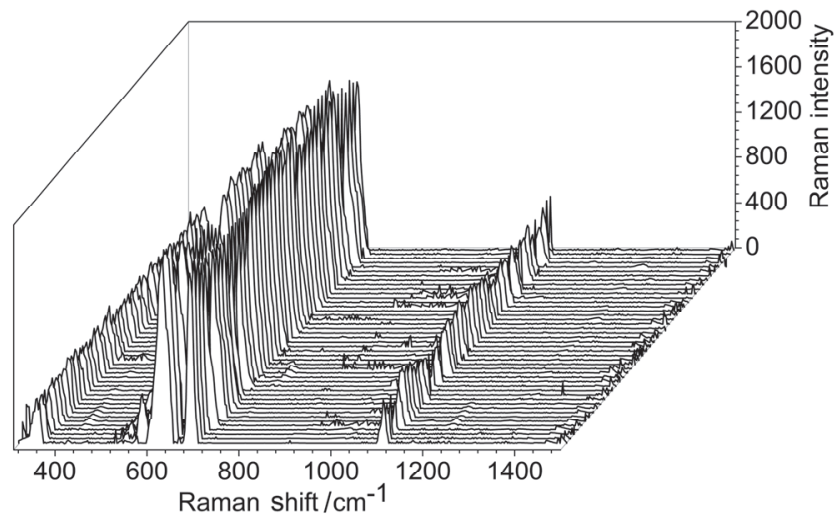

图 4 相同条件下 50 组样品的 SERS 谱图

Figure 4 SERS spectra of 50 samples under same conditions

后的光谱曲线, 图 $5 \mathrm{c}$ 为 MSAZ 处理后的光谱曲线. 两种 光谱预处理方法均可有效扣除苂光背景, 特别对于峰值 较小的拉曼峰( $348,535,568 \mathrm{~cm}^{-1}$ 等), 经过预处理后, 可得到有效的提取, 较为全面地保留毒死蜱农药的信 息.

\section{5 农药拉曼光谱定量分析}

对苹果样品进行定量分析之前, 首先对毒死蜱标准 溶液进行 SERS 检测, 图 2s 为不同浓度的毒死蜱标准溶 液的 SERS 光谱曲线, 所检测的毒死蜱溶液浓度范围为 $1 \times 10^{-4} \sim 1.25 \mathrm{mg} / \mathrm{L}$, 当农药浓度为 $1 \times 10^{-3} \mathrm{mg} / \mathrm{L}$ 时, 毒死蜱的特征峰 $\left(621 \mathrm{~cm}^{-1} 、 680 \mathrm{~cm}^{-1}\right)$ 可以清晰辨识, 当 浓度进一步降低, 毒死蜱的特征峰强度较弱, 很难辨识. 因此采用该方法，可以检测出浓度高于 $1 \times 10^{-3} \mathrm{mg} / \mathrm{L}$ 的 毒死蜱. 

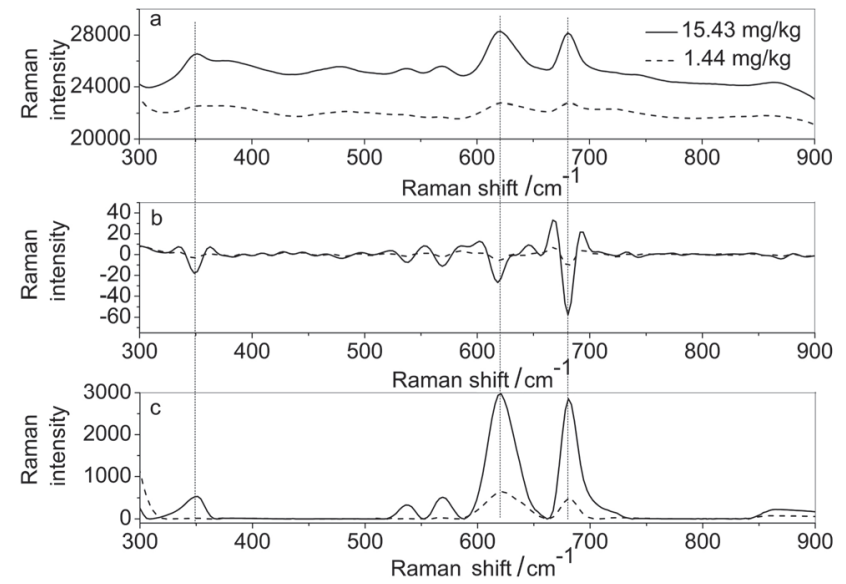

图 5 原始光谱图(a)和经过 SD (b)、MSAZ (c) 预处理后的光谱图

Figure 5 Representative SERS spectra without treatment (a) and with $\mathrm{SD}$ (b) and MSAZ (c) treatments

将苹果样品在不同浓度的毒死蜱中浸泡后晾干, 毒 死蜱农药基本存在于苹果表皮(实际样品中苹果农药残 留也集中于苹果表皮 ${ }^{[24,25]}$, 对样品进行多点拉曼扫描, 平均光谱作为每个苹果样品的拉曼图谱. 采集光谱后, 利用气相色谱法检测每个苹果样品的农药含量, 其毒死 蜱含量范围为 $15.52 \sim 0.02 \mathrm{mg} / \mathrm{kg}$. 图 6a 和图 $6 \mathrm{~b}$ 分别为 经过 MSAZ 和 SD 前处理后 12 个不同农药含量的苹果 的拉曼光谱图. 图 6a 中, 当苹果农药含量为 15.43 $\mathrm{mg} / \mathrm{kg}$ 时，毒死蜱在 348、535、568、621、680、1099 $\mathrm{cm}^{-1}$ 处的特征峰能够明显分辨, 随着农药含量的下降, 各特征峰强度逐渐降低, 当苹果的毒死蜱含量低于 1.44 $\mathrm{mg} / \mathrm{kg}$ 时, 348、535、568 和 $1099 \mathrm{~cm}^{-1}$ 处的特征峰基本 消失, 但是 621 和 $680 \mathrm{~cm}^{-1}$ 处的特征峰仍旧清晰可见, 甚至农药浓度下降为 $0.064 \mathrm{mg} / \mathrm{kg}$ 时, 仍可分辨出 621 和 $680 \mathrm{~cm}^{-1}$ 处的拉曼峰. 图 6b 中, 对原始光谱图经过二 阶导后，毒死蜱特征峰得到有效的提取，以 621 和 680 $\mathrm{cm}^{-1}$ 为例, 两个峰高随着毒死蜱浓度减小均逐渐减小, 当毒死蜱浓度较低时, 其特征峰仍明显可辨.

\section{6 拉曼光谱定量模型的建立}

通过建立数学模型能进一步了解拉曼光谱与样品 毒死蜱含量的关系. 并可通过比较校正集的相关系数 (Correlation Coefficient of Calibration set, $R_{\mathrm{c}}$ )与预测集的 相关系数 (Correlation Coefficient of Prediction set, $R_{\mathrm{p}}$ )、校 正均方根误差(Root Mean Square Errors of Calibration, RMSEC)和预测均方根误差(Root Mean Square Error of Predictions, RMSEP)来评价模型.

经过 SD 前处理后, 分别建立样品毒死蜱含量与 621 和 $680 \mathrm{~cm}^{-1}$ 处峰强度的一元线性回归模型, 并建立 毒死蜱含量与 621 和 $680 \mathrm{~cm}^{-1}$ 处的峰强度的二元线性回 归模型; 经过 MSAZ 预处理后, 分别建立毒死蜱含量与 621 和 $680 \mathrm{~cm}^{-1}$ 处峰面积及峰强度的一元线性回归模 型, 并分别建立毒死蜱含量与 621 和 $680 \mathrm{~cm}^{-1}$ 处的峰强
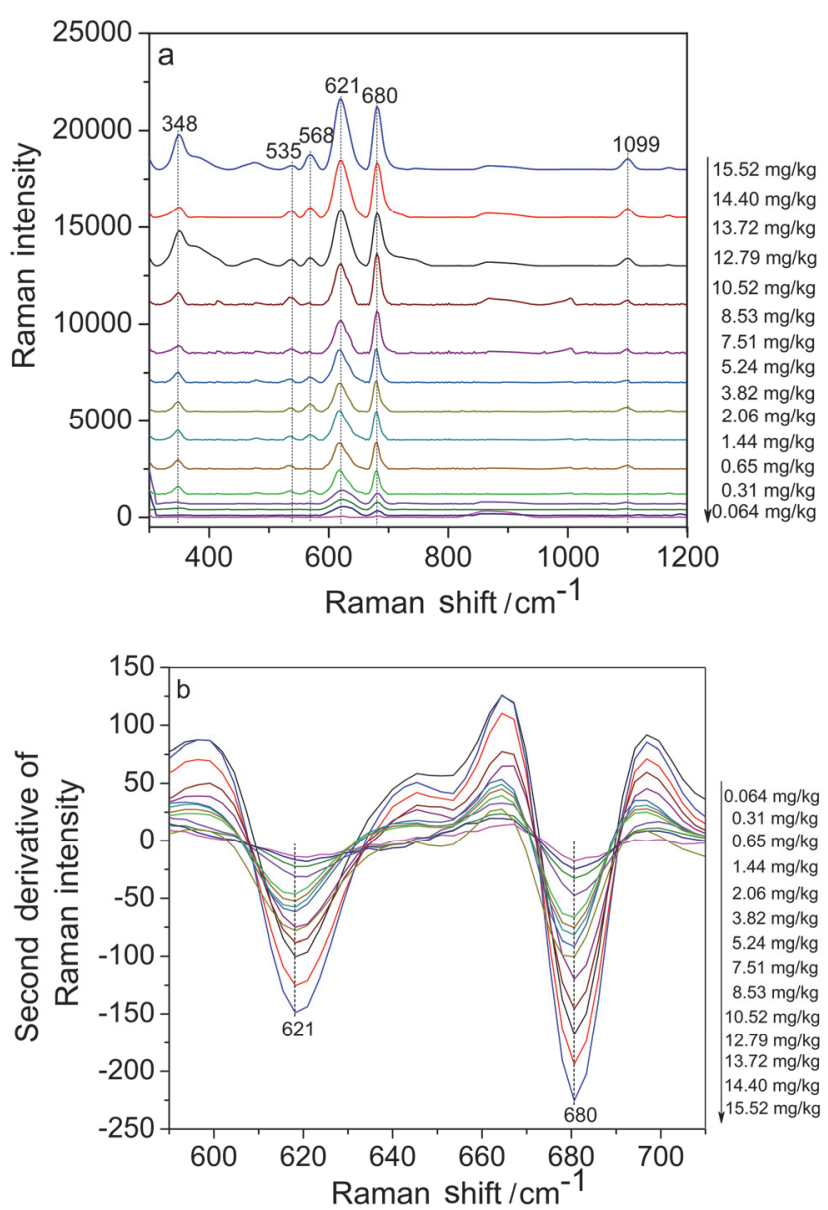

图 6 MSAZ 预处理后(a)和 SD 预处理后(b)的不同毒死蜱浓度的苹果 SERS 平均光谱图 $(n=30)$

Figure 6 Average SERS spectra $(n=30)$ of chlorpyrifos at different concentrations after MSAZ (a) and SD (b)

度及峰面积的二元线性回归模型, 结果见表 1. 九种建 模方法中预测集的相关系数均大于 0.85 , 其中, 光谱经 过 MSAZ 预处理后, 以峰强度作为自变量建立的二元线 性回归模型, 其预测集相关系数达到 0.969 , 具有较高 的预测精度. 图 7 为采用该建模方法时，毒死蜱校正集 和预测集的毒死蜱含量真实值与预测值的散点分布图.

表 1 不同预处理方法建立 MLR 模型的校正集和预测集结果

Table 1 Calibration and prediction results of chlorpyrifos concentrations by using MLR method

\begin{tabular}{ccccccc}
\hline 前处理方法 变量 & 拉曼峰 $/ \mathrm{cm}^{-1}$ & $R_{\mathrm{c}}$ & RMSEC & $R_{\mathrm{p}}$ & $\mathrm{RMSEP}$ \\
\hline \multirow{4}{*}{$\mathrm{SD}$} & & 621 & 0.905 & 1.33 & 0.887 & 1.43 \\
& 峰强度 & 680 & 0.916 & 1.31 & 0.896 & 1.49 \\
& & 621 和 680 & 0.921 & 1.30 & 0.903 & 1.42 \\
\cline { 3 - 7 } MSAZ & 621 & 0.926 & 1.34 & 0.911 & 1.35 \\
& 峰面积 & 680 & 0.929 & 1.29 & 0.916 & 1.31 \\
& & 621 和 680 & 0.953 & 1.28 & 0.915 & 1.33 \\
\cline { 2 - 7 } & 621 & 0.966 & 1.18 & 0.954 & 1.29 \\
& 峰强度 & 680 & 0.970 & 1.15 & 0.960 & 1.34 \\
& & $\mathbf{6 2 1}$ 和 $\mathbf{6 8 0}$ & $\mathbf{0 . 9 7 2}$ & $\mathbf{1 . 1 9}$ & $\mathbf{0 . 9 6 9}$ & $\mathbf{1 . 2 4}$ \\
\hline
\end{tabular}



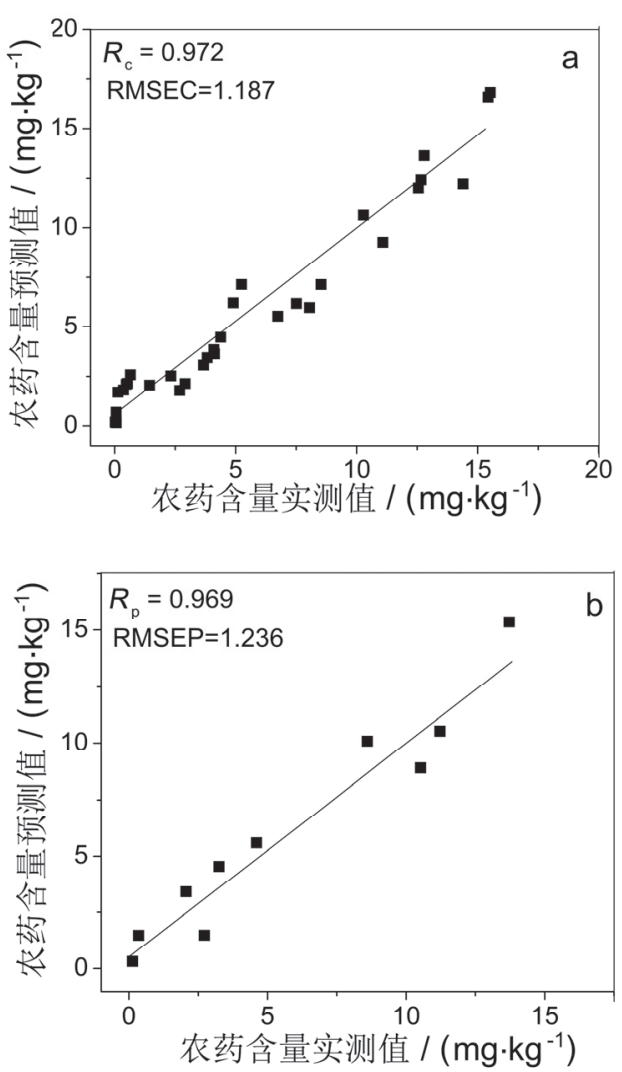

图 7 毒死蜱含量校正集(a)和预测集(b)的最佳预测结果

Figure 7 Optimum calibration (a) and prediction (b) results of chlorpyrifos concentrations

\section{3 结论}

利用实验室自行搭建的拉曼系统, 研究了银溶胶作 为表面增强剂的效果, 探讨了丙酮和硝酸溶液对增强效 果的影响, 调查了银溶胶的储存稳定性以及实验方法和 光谱系统的实验稳定性, 建立了毒死蜱含量与特征峰强 度的线性回归模型. 结果表明, 当苹果的毒死蜱含量为 $0.064 \mathrm{mg} / \mathrm{kg}$ 时, 仍可以观察到明显的拉曼特征峰, 以该 方法检测样品的农药含量具有较高的稳定性, 最优条件 下建立的预测模型的相关系数为 0.969 . 本研究采用的 表面增强方法进一步降低了水果中农药含量的检测限, 建立了完整苹果中农药残留的预测模型, 这为水果的安 全检测提供了一种快速灵敏的检测方法. 然而, 要保证 模型的适用性和稳定性, 待检测的苹果样品需要满足合 适的质量范围, 在检测此范围外的苹果样品时, 如何结 合苹果质量、直径和表面积等参数对模型进行修正仍需 进一步研究.

\section{4 实验部分}

\section{1 样品制备}

对从超市购买的新鲜红富士苹果进行比表面积检 测, 选取质量在 $150 \sim 240 \mathrm{~g}$ 范围内的苹果作为测试样 品, 此范围内的苹果其比表面积基本恒定, RSD 约为
$4.8 \%$. 将苹果仔细冲洗干净，待表面水分完全挥发后使 用. 以丙酮作为溶剂, 将毒死蜱乳油制备成浓度范围为 48 4800 mg/L 的溶液(注，毒死蜱用于防治苹果树中的 棉蚜，一般施用浓度为 $200 \sim 267 \mathrm{mg} / \mathrm{L}$ )，搅拌均匀后， 将干净的苹果浸入农药中, $5 \mathrm{~s}$ 后取出, 在室温下自然干 燥 $8 \mathrm{~h}$

\section{2 银溶胶的制备}

根据 Leopold 等的方法 ${ }^{[26]}$, 首先制备 $1.11 \times 10^{-3}$ $\mathrm{mol} / \mathrm{L}$ 的硝酸银溶液, 然后将盐酸差弪胺和氢氧化钠溶解 于 $10 \mathrm{~mL}$ 的去离子水中, 浓度分别为 $1.5 \times 10^{-2} \mathrm{~mol} / \mathrm{L}$ 和 $3 \times 10^{-2} \mathrm{~mol} / \mathrm{L}$, 将新鲜制备的混合液迅速倒入 $90 \mathrm{~mL}$ 硝 酸银溶液中，混合均匀，溶液颜色由透明变为灰褐色， 持续摚拌 $30 \mathrm{~min}$ 即得到纳米银胶, 将制备好的银溶胶置 于 $4{ }^{\circ} \mathrm{C}$ 黑暗环境中待用.

\section{3 拉曼光谱的采集}

采集拉曼光谱前, 先在苹果表面滴涂 $2 \mu \mathrm{L}$ 丙酮溶 液(散开直径 $2 \mathrm{~mm}$ ), 然后迅速滴加 $4 \mu \mathrm{L}$ 经过离心浓缩 后的银溶胶(散开直径 $3 \mathrm{~mm}$ ), 最后滴加 $1 \mu \mathrm{L} 40 \mathrm{mmol} / \mathrm{L}$ 的硝酸溶液(散开直径 $3 \mathrm{~mm}$ ), 反应 $20 \mathrm{~s}$ 后进行拉曼信号 采集, 拉曼光谱采集参数设置为: 光谱范围 -176 $2400 \mathrm{~cm}^{-1}$, 分辨率 $2 \mathrm{~cm}^{-1}$, 激光波长 $785 \mathrm{~nm}$, 激光功率 $450 \mathrm{~mW}$, 曝光时间 $3 \mathrm{~s}$. 为了能够准确得到苹果样品的 拉曼信息, 每个样品采集 30 个点, 采集点的位置分散于 样品的整个表面, 每个点分别滴涂丙酮、银溶胶和硝酸 溶液, 光谱采集后, 对 30 条光谱进行平均, 得到的光谱 曲线作为样品原始光谱. 按照 $3: 1$ 的比例将样品分为 校正集和预测集, 31 个苹果样品为校正集, 10 个苹果样 品作为预测集.

\section{4 气相方法检测苹果农药含量}

将采集光谱后的苹果迅速放入密封袋中保存, 参照 标准方法 SN/T 0148-2011，采用气相色谱法对苹果中毒 死蜱含量进行检测.

\section{References}

[1] Li, R.; He, L.; Wei, W.; Hao, L.; Ji, X.; Zhou, Y.; Wang, Q. Food Control 2015, 51, 212.

[2] Kim, Y. A.; Lee, E. H.; Kim, K. O.; Lee, Y. T.; Hammock, B. D.; Lee, H. S. Anal. Chim. Acta 2011, 693, 106.

[3] Boeris, V.; Arancibia, J. A.; Olivieri, A. C. Anal. Chim. Acta 2014 $814,23$.

[4] Garcia-Rodriguez, D.; Cela-Torrijos, R.; Lorenzo-Ferreira, R. A.; Carro-Diaz, A. M. Food Chem. 2012, 135, 259.

[5] Zhang, Z.; Liu, R.; Xu, D.; Liu, J. Acta Chim. Sinica 2012, 70, 1686 (in Chinese). (张宗绵, 刘睿, 徐敦明, 刘景富, 化学学报, 2012 70, 1686.)

[6] Wang, X.; Du, Y.; Zhang, H.; Xu, Y.; Pan, Y.; Wu, T.; Hu, H. Food Control 2014, 46, 108.

[7] Li, X.; Gao, G.; Niu, L.; Lin, M.; Qin, Z.; Liu, J.; Yao, H. Chinese J. Anal. Chem. 2012, 40, 1494 (in Chinese). (李雪, 高国明, 牛丽媛, 林漫漫，覃宗定，刘军贤，姚辉璐，分析化学, 2012,40,1494.)

[8] Ren, B.; Tian, Z. Modern Instruments 2004, 10, 1 (in Chinese). (任 斌, 田中群, 现代仪器, 2004, 10,1.)

[9] Li, C.; Lai, K.; Zhang, Y.; Pei, L.; Huang, Y. Acta Chim. Sinica 2013, 71, 221 (in Chinese). (李春渘, 赖克强, 张源园, 裴鹭, 黄轶 群, 化学学报, 2013, 71, 221.) 
[10] Chen, X.; Zhao, A.; Gao, Q.; Gan, Z.; Tao, W. Acta Chim. Sinica 2014, 72, 467 (in Chinese). (陈旭成, 赵爱武, 高倩, 甘自保, 陶文 玉, 化学学报, 2014, 72, 467.)

[11] Stiufiuc, R.; Iacovita, C.; Lucaciu, C. M.; Stiufiuc, G.; Dutu, A. G.; Braescu, C.; Leopold, N. Nanoscale Res. Lett. 2013, 8, 47.

[12] Muller, C.; David, L.; Chis, V.; Pinzaru, S. C. Food Chem. 2014, 145,814 .

[13] He, L.; Chen, T.; Labuza, T. P. Food Chem. 2014, 148, 42.

[14] Xie, Y.; Mukamurezi, G.; Sun, Y.; Wang, H.; Qian, H.; Yao, W. Eur. Food Res. Technol. 2012, 234, 1091.

[15] Dhakal, S.; Li, Y.; Peng, Y.; Chao, K.; Qin, J.; Guo, L. J. Food Eng. 2014, 123, 94

[16] Gao, G.; Li, X.; Qin, Z.; Wei, K.; Huang, H.; Liu, J.; Yao, H. Acta Optica Sinica 2013, 33, 0230002-1 (in Chinese). (高国明, 李雪, 覃 宗定, 魏坤莲, 黄汉明, 刘军贤, 姚辉璐, 光学学报, 2013, 33, 0230002-1.)

[17] Canamares, M. V.; Garcia-Ramos, J. V.; Gomez-Varga, J. D.; Domingo, C.; Sanchez-Cortes, S. Langmuir 2005, 21, 8546.

[18] Kang, Y.; Wu, T.; Liu, B.; Wang, X.; Du, Y. Microchim. Acta 2014, $181,1333$.
[19] Jana, N. R.; Pal, T. Adv. Mater. 2007, 19, 1761.

[20] Si, M.; Zhang, P. Spectroscopy and Spectral Analysis 2001, 21, 501 (in Chinese). (司民真, 张鹏翔, 光谱学与光谱分析, 2001, 21, 501.)

[21] Li, D.; Cao, B.; Yang, G.; Li, Z.; Gao, S.; Zhou, M.; Men, Z.; Zhang, X. The Journal of Light Scattering 2009, 21, 132 (in Chinese). (李东 飞, 曹彪, 杨光, 里佐威, 高淑琴, 周密, 门志伟, 张学亚, 光散 射学报, 2009, 21, 132.)

[22] Shende, C.; Inscore, F.; Sengupta, A.; Stuart, J.; Farquharson, S. Sens. Instrum. Food Qual. Saf. 2010, 4, 101.

[23] Fan, Y. X.; Lai, K. Q.; Rasco, B. A.; Huang, Y. Q. Food Control 2014, 37, 153.

[24] Chen, Z.; Chen, J.; Han, M.; Wang, W.; Cao, W. J. Agro-Environ. Sci. 2011, 30, 2197 (in Chinese). (陈振德，陈建美，韩明三，王文 娇，曹委，农业环境科学学报, 2011, 30, 2197.)

[25] Zhang, X. M.S. Thesis, North West Agriculture and Forestry University, Yangling, 2007 (in Chinese). (张晓荣, 硕士论文, 西北农林科 技大学, 杨凌, 2007.)

[26] Leopold, N.; Lendl, B. J. Phys. Chem. B 2003, 107, 5723.

(Cheng, B.) 